\title{
The Mass-Energy Equivalence Principle in Fluid Dynamics
}

\author{
Angel Fierros Palacios \\ Instituto de Investigaciones Eléctricas, División de Energías Alternas, Mexico City, México \\ Email: afierros@iie.org.mx
}

Received 4 June 2015; accepted 25 July 2015; published 28 July 2015

Copyright (C) 2015 by author and Scientific Research Publishing Inc.

This work is licensed under the Creative Commons Attribution International License (CC BY). http://creativecommons.org/licenses/by/4.0/

(c) () Open Access

\begin{abstract}
From Lagrangian formalism as in Classical Field Theory and within the theoretical scheme of the Hamilton-Type Variational Principle, the mass-energy equivalence principle for any fluid is obtained.
\end{abstract}

Keywords

The Mass-Energy Equivalence Principle, Fluid Dynamics

\section{Introduction}

In the relativistic formulation of particle mechanics, it is demonstrated that the energy of a free particle does not vanish when its speed goes to zero. Instead, it reaches a finite value called the energy at rest of the particle. This is one of the best known, spectacular and important results of the Special Theory of Relativity of A. Einstein. The $E=m c^{2}$ Equation expresses the fact that mass and energy are equivalent, that is, they conform a single invariant denominated the mass-energy relation. It has been confirmed through multiple practical applications and according to Einstein its validity extends to the whole Universe. As a consequence, it is natural to state that this result can also be derived from the Hamilton-Type Principle of Fluid Mechanics within the theoretical scheme of Lagrange’s Analytical Mechanics.

\section{The Mass-Energy Relation}

Consider any fluid confined within an arbitrary region $R$ of the three-dimensional Euclidian space. It is desired to obtain the mass-energy equivalence principle for this continuous system with the help of the Lagrange formalism. In the analytical treatment of fluid dynamics [1], an action functional which is a space-time integral 


$$
W=\int_{t_{1}}^{t_{2}} \int_{R} \ell \mathrm{d} V \mathrm{~d} t
$$

of a Lagrangian density

$$
\ell=\ell(J ; \overrightarrow{\operatorname{grad}} J)
$$

is commonly used. In this case the Lagrangian density is a function of the Jacobian $J$ and its first gradient [1] [2]; which implies that it is a geometric Lagrangian density. According to the Hamilton-Type Principle, the action integral remains invariant for continuous infinitesimal geometric variations with respect to a set of continuous time independent geometric parameters $\{\alpha\}$, from which the field variables and coordinates depend; that is [1]

$$
\delta W=\frac{\partial W}{\partial \alpha} \delta \alpha=0
$$

Furthermore, the following boundary condition of general character is imposed on the coordinates

$$
\delta x^{i}\left(t_{1}\right)=\delta x^{i}\left(t_{2}\right)=0 .
$$

It can be shown that the local variation of the action integral (1) and the application of the Hamilton-Type Principle provide as a direct consequence the following result [1]

$$
\int_{t_{1}}^{t_{2}} \int_{R} \rho \delta \lambda \mathrm{d} V \mathrm{~d} t=0
$$

where

$$
\lambda=\lambda(J ; \operatorname{grad} J)
$$

is the specific Lagrangian. The Lagrangians (2) and (6) are related as follows [3] [4]

$$
\ell=\rho \lambda
$$

where $\rho(x, t)$ is the mass density. According to the functionality of $\lambda$ given in (6) is it evident that

$$
\rho \delta \lambda=\rho \frac{\partial \lambda}{\partial J} \delta J+\rho \frac{\partial \lambda}{\partial \nabla J} \delta \nabla J
$$

It can be demonstrated that [1]

$$
\delta J=J \operatorname{div}(\delta x)
$$

and

$$
\delta \nabla J=\operatorname{grad} J \frac{\partial}{\partial x^{i}}\left(\delta x^{i}\right)
$$

In that case, the following result can be obtained from (8):

$$
\rho \delta \lambda=\frac{\partial}{\partial x^{i}}\left[\rho\left(J \frac{\partial \lambda}{\partial J}+\nabla J \frac{\partial \lambda}{\partial \nabla J}\right) \delta x^{i}\right]-\frac{\partial}{\partial x^{i}}\left[\rho\left(J \frac{\partial \lambda}{\partial J}+\nabla J \frac{\partial \lambda}{\partial \nabla J}\right)\right] \delta x^{i} ;
$$

where integration by parts has been performed. If the first term of the right hand side of (10) is used in (5) and the Green's theorem is applied, the following result is obtained

$$
\int_{t_{1}}^{t_{2}} \int_{S} \rho\left[J \frac{\partial \lambda}{\partial J}+\nabla J \frac{\partial \lambda}{\partial \nabla J}\right] \delta x^{i} \mathrm{~d} a_{i} \mathrm{~d} t
$$

where $S$ is the surrounding area of region $R$ and $\mathrm{d} a$ is the differential of area. The surface integral is null due to 
the following. Consider a continuous medium contained within a region $R$ which is not deformed at infinity and the integration surface extended to infinity, where $J=1$, in such a way that $\nabla J=0$ and the integral vanishes. The substitution of the second term of the right hand side of (10) into (5) provides the following result

$$
\int_{t_{1}}^{t_{2}} \int_{R}\left\{\frac{\partial}{\partial x^{i}}\left[\rho\left(J \frac{\partial \lambda}{\partial J}+\nabla J \frac{\partial \lambda}{\partial \nabla J}\right)\right]\right\} \delta x^{i} \mathrm{~d} V \mathrm{~d} t=0 .
$$

As the local variations of $x$ are arbitrary and linearly independent among them, and $\mathrm{d} V$ as well as $\mathrm{d} t$ are completely arbitrary increments and therefore different from zero, the previous equation is satisfied only if the integrand vanishes; that is

$$
\rho \operatorname{grad}\left[J \frac{\partial \lambda}{\partial J}+\nabla J \frac{\partial \lambda}{\partial \nabla J}\right]+\left[J \frac{\partial \lambda}{\partial J}+\nabla J \frac{\partial \lambda}{\partial \nabla J}\right] \operatorname{grad} \rho=0
$$

where the derivative has been calculated. Nevertheless, it can be seen in the first term of (13), that

$$
\rho\left[\frac{\partial J}{\partial x^{i}} \frac{\partial \lambda}{\partial J}+\frac{\partial \nabla J}{\partial x^{i}} \frac{\partial \lambda}{\partial \nabla J}\right]=2 \rho \frac{\partial \lambda}{\partial x^{i}}=0 ;
$$

where the chain rule has been used to obtain the result. The term is zero because $\lambda, \partial \lambda / \partial J$ and $\partial \lambda / \partial \nabla J$ are not explicit functions of $x$. As grad $\rho \neq 0$, it follows from (13) that

$$
\frac{\partial \lambda}{\partial J}+\frac{1}{J} \frac{\partial \lambda}{\partial \nabla J} \operatorname{grad} J=0 .
$$

This is the field differential equation for the mass density in terms of the specific lagrangian. Let

$$
\lambda=\varepsilon_{o}\left[J \Gamma_{i k}^{i} u_{k}+u_{k} \frac{\partial J}{\partial x^{k}}\right]
$$

be the explicit form of the specific Lagrangian [1] [2]. Where, $\varepsilon_{0}$ is the constant equilibrium value of the internal specific energy, $\Gamma_{i k}^{i}=\Gamma_{k i}^{i}$ is the contracted Christoffel symbol and $u(x)$ is the displacement vector [1] [5] [6]. Hence, using (16) in (15) produces

$$
\rho(x, t)=\frac{\rho_{o}}{J} u \cdot \operatorname{grad} J
$$

as by definition [1] [2]

$$
\Gamma_{i k}^{i} u_{k}=-\frac{\rho(x, t)}{\rho_{o}} .
$$

Equation (17) is the scalar equation for the mass density [1] [2]. On the other hand, within the theoretical scheme of the Hamilton-Type Variational Principle, it is demonstrated that the action integral (1) remains invariant to an infinitesimal continuous transformation with respect to time; thus,

$$
\delta^{+} W=0
$$

with

$$
\delta^{+}() \equiv \frac{\mathrm{d}}{\mathrm{d} t}() \delta^{+} t
$$

being the time variation definition [1]. Besides, the temporary variations are subject to the following general boundary condition [1]

$$
\delta^{+} t_{1}=\delta^{+} t_{2}=0 .
$$

Hence, the invariance condition (19) applied to the action integral (1) provides the following as a general result 


\section{[1] [4]}

$$
\int_{t_{1}}^{t_{2}} \int_{R}\left(\delta^{+} \ell-\frac{\mathrm{d} \ell}{\mathrm{d} t} \delta^{+} t\right) \mathrm{d} V \mathrm{~d} t=0 .
$$

According to the functionality of $\ell$ given in (2)

$$
\delta^{+} \ell=\frac{\partial \ell}{\partial J} \delta^{+} J+\frac{\partial \ell}{\partial \nabla J} \delta^{+} \nabla J .
$$

Besides and by the definition in (20), it follows that

$$
\delta^{+} J=\frac{\mathrm{d} J}{\mathrm{~d} t} \delta^{+} t=J \operatorname{div} v \delta^{+} t ;
$$

where the Euler relation [1]

$$
\frac{\mathrm{d} J}{\mathrm{~d} t}=J \operatorname{div} v,
$$

has been considered. Moreover, it is clear that [1]

$$
\delta^{+}(\operatorname{grad} J)=(\operatorname{grad} J) \operatorname{div} v \delta^{+} t ;
$$

and hence

$$
\delta^{+} \ell=\left[\frac{\partial \lambda}{\partial J}+\frac{1}{J} \frac{\partial \lambda}{\partial \nabla J} \operatorname{grad} J\right] \rho J \operatorname{div} v \delta^{+} t .
$$

In that case $\delta^{+} \ell=0$, because the field Equation (15) is contained within the square bracket, and that term is zero. Therefore, (22) becomes

$$
\int_{t_{1}}^{t_{2}} \int_{R} \frac{\mathrm{d} \ell}{\mathrm{d} t} \delta^{+} t \mathrm{~d} V \mathrm{~d} t=0,
$$

which is satisfied only if the integrand vanishes [1]. Indeed, as the temporary variations $\delta^{+} t$ are arbitrary and linearly independent among them, and $\mathrm{d} V$ as well as $\mathrm{d} t$ are arbitrary increments and therefore different from zero, it follows that

$$
\frac{\mathrm{d} \ell}{\mathrm{d} t}=0
$$

which is the mass balance equation. Effectively, it can be demonstrated (see Appendix) that the relativistic lagrangian density for any fluid free of forces is

$$
\ell=-\rho c^{2} \sqrt{1-\frac{v^{2}}{c^{2}}}
$$

In the case of a fluid at rest, $\ell=-\rho c^{2}$, and according to (7), $\lambda=-c^{2}$; in such a way that neither $\ell$, and of course nor $\lambda$ are functions of the velocity field; so that

$$
\frac{\mathrm{d} \ell}{\mathrm{d} t}=\rho c^{2} \operatorname{div} v .
$$

From the hydrodynamics derivative definition [7] and taking in to account the relationship (7) it is easy to obtain that

$$
\frac{\mathrm{d} \ell}{\mathrm{d} t}=\lambda\left[\frac{\partial \rho}{\partial t}+\operatorname{div}(\rho v)\right]+\rho \frac{\partial \lambda}{\partial t}-\lambda \rho \operatorname{div} v
$$


where an integration by parts was made, and it was considered that $\lambda$ is not an explicit function of $x$. Finally, from (29) and (30), the following result is obtained

$$
\lambda\left[\frac{\partial \rho}{\partial t}+\operatorname{div}(\rho v)\right]+\rho \frac{\partial \lambda}{\partial t}=0 .
$$

Time uniformity has as a consequence that the specific Lagrangian does not become an explicit function of time; so that $\partial \lambda / \partial t=0$, and then in (31) we have that

$$
\frac{\partial \rho}{\partial t}+\operatorname{div}(\rho v)=0
$$

because $\lambda \neq 0$. This is the mass balance equation.

Furthermore, the invariance of the action under transformations with respect to the evolution parameter and due to the uniformity of time, more than the mass balance equation, the energy balance equation should be obtained from Equation (28). As a consequence from the obtained result, it seems natural to assume that in the field of fluid dynamics there is a close relationship between the densities of mass and energy of any continuous medium. To prove the previous assumption, consider the general definition of the hamiltonian density [1] [4]

$$
\mathcal{H}=\frac{\partial \ell}{\partial v^{i}} v^{i}-\ell ;
$$

here $v^{i}$ is the $i$ th-component of the velocity field. As for the present case the geometric lagrangian density (2) does not depend on the velocity field, it holds that $\mathcal{H}=-\ell$ and therefore from (28) the following result can be obtained

$$
\mathcal{H}=\text { constant } .
$$

Assume that

$$
\mathcal{H}=\rho \beta^{2},
$$

with $\rho$ again as the mass density and $\beta^{2}$ as a constant with units of velocity squared that has the purpose of balancing dimensions in Equation (35). It will be shown in the appendix that $\beta^{2}=c^{2}$, being $c$ the velocity of light in vacuum space. Furthermore

$$
\rho=\frac{\rho_{o}}{\sqrt{1-v^{2} / c^{2}}}
$$

where $\rho_{o}$ is the mass density at rest and $v$ the magnitude of the flow velocity. Using the previous definitions, it follows that

$$
\mathcal{H}=\frac{\rho_{o} c^{2}}{\sqrt{1-v^{2} / c^{2}}} ;
$$

and the condition imposed in (34) is satisfied because the flow rate is constant. The previous equation shows that in the domain of relativistic dynamics, the kinetic energy of a fluid does not vanish when its flow rate vanishes, since for such case $\mathcal{H}$ has the following value as it can be seen from (37)

$$
\mathcal{H}=\rho_{o} c^{2} .
$$

This is the equation for the energy density of a continuous at rest medium. It expresses the equivalence between energy and mass densities in the field of fluid dynamics. It is clear that as $\mathcal{H}$ is the energy per unit volume and $\rho_{o}$ the mass of fluid also per unit volume, Equation (38) reduces to the well known Einstein Equation of relativistic mechanics, $E=m_{o} c^{2}$; except that here $m_{o}$ is not the mass of a free particle at rest, but the mass of the continuous medium at rest, free of forces.

Furthermore, for low flow rates when $v / c \ll 1$, Equation (37) can be expanded into a power series of $v / c$ to obtain 


$$
\mathcal{H} \approx \rho c^{2}+\frac{1}{2} \rho v^{2} .
$$

The form of the previous equation is due to the fact that when $v / c \rightarrow 0, \rho \rightarrow \rho_{o}$. This is the Newtonian limit of the general equation for the relativistic mass density $\rho$. Except for the energy at rest, the previous equation is the expression for the kinetic energy density of the continuous medium under study. It is clear from Equation (36) that the relativistic relationship between mass and velocity is satisfied [8]; that is

$$
m=\frac{m_{o}}{\sqrt{1-v^{2} / c^{2}}},
$$

where $m_{o}$ is the mass of the fluid at rest and $v$ the magnitude of the flow velocity. Finally, from the total derivative of Equation (35) with respect to time and as $\rho \beta^{2} \neq 0$, it can be directly obtained that

$$
\operatorname{div} v=0 .
$$

This is the continuity equation for the case in which the mass density is referred to the system at rest. The previous relation is identically satisfied because the system under consideration is not in motion, and so the flow velocity is zero. In addition, as the kind of fluid contained in $R$ has not been specified anywhere, the massenergy relation is valid for any continuous medium, and so are the rest of the results obtained.

\section{Conclusions}

The theoretical scheme of the Hamilton-Type Variational Principle provides the methodology required to obtain the mass-energy equivalence principle for any fluid. The problem is enclosed within the Lagrange formulation of theoretical mechanics, in such a way that the required methodology is configured with the help of the action integral and a Lagrangian density depending only on purely geometric entities. The local variation of the action integral and the usage of appropriate boundary conditions within the Hamilton-Type Principle scheme produce as a result of the field equation for the mass density. The scalar equation for the mass density is obtained using the explicit form of the specific Lagrangian. As a result of the temporary variation of the action integral, the referred Hamilton-Type Principle and the corresponding boundary conditions, the mass balance equation is obtained.

Finally, from the mass balance equation and the general definition of the Hamiltonian density, the equivalence principle between the mass and energy densities in fluid dynamics is obtained. Furthermore, the relationship between the mass density and the flow velocity is provided, and the corresponding continuity equation for the case of mass density referred to a continuous medium at rest is obtained.

\section{References}

[1] Fierros Palacios, A. (2006) El Principio tipo Hamilton en la Dinámica de los Fluidos, primera y segunda ediciones. McGraw-Hill Interamericana Editores, S.A. de C.V. (1998 y 1999). México. Buenos Aires. Madrid. Nueva York. Montreal. Sidney. etc. The Hamilton-Type Principle in Fluid Dynamics. Fundamentals and Applications to Magnetohydrodynamics, Thermodynamics, and Astrophysics.Springer-Verlag. Wien.

[2] Fierros Palacios, A. (1994) La ecuación de campo para la densidad de masa. Rev. del IMP, XXIV.

[3] Viniegra, H.F., Salcido, G.A. and Fierros Palacios, A. (1979) Las ecuaciones de balance de un fluido perfecto a partir de un principio variacional tipo Hamilton. Rev. del IMP, XI.

[4] Fierros Palacios, A. (1992) Las ecuaciones de balance para un fluido viscoso a partir de un principio variacional tipo Hamilton. Rev. Mex. Fis., 38, 4518-4531.

[5] Landau, L.D. and Lifshitz, E.M. (1959) Theory of Elasticity. Addison-Wesley Publishing Co., Boston.

[6] Landau, L.D. and Lifshitz, E.M. (1962) The Classical Theory of Fields. Addison-Wesley Publishing Co., Boston.

[7] Landau, L.D. and Lifshitz, E.M. (1959) Fluid Mechanics. Addison-Wesley Publishing. Co., London, Paris, Frankfurt.

[8] Resnick, R. (1976) Conceptos de relatividad yteoría cuántica. Editorial Limusa, México. 


\section{Appendix}

Let $R$ be a region in the three-dimensional Euclidian space containing any fluid. Let $\ell$ be the lagrangian density such that the classic lagrangian has the following value

$$
L=\int_{R} \ell \mathrm{d} V
$$

where $\mathrm{d} V$ is the volume element. According to relativistic dynamics, the classic lagrangian for a free particle [6] of mass $m$ in motion with a constant velocity $v$ is

$$
L=-m c^{2} \sqrt{1-\frac{v^{2}}{c^{2}}} .
$$

Never the less, the previous relationship also holds for an extended body; in particular, it must be valid for any continuous medium whose total mass is given by

$$
m=\int_{R} \rho(x, t) \mathrm{d} V ;
$$

where $\rho(x, t)$ is the mass density. Substituting (A-3) in (A-2) and comparing the result with (A-1), it follows that

$$
\ell=-\rho c^{2} \sqrt{1-\frac{v^{2}}{c^{2}}} .
$$

In the newtonian limit of relativistic mechanics; that is, when $v / c \rightarrow 0$, (A-4) yields $\ell \rightarrow-\rho c^{2}$. In that case and since $H=-\ell, \beta^{2}=c^{2}$, the assumption made after Equation (35) is satisfied. Besides, according to the Lorentz contraction [6] the proper volume of the continuous medium under consideration is

$$
V=V_{o} \sqrt{1-\frac{v^{2}}{c^{2}}} .
$$

Since $V=m / \rho$ and $V_{o}=m / \rho_{o}$, Equation (36) is satisfied. 